\title{
Psicologia E INCLUSÃo ESCOLAR: NOVAS POSSIBILIDADES DE INTERVIR PREVENTIVAMENTE SOBRE PROBLEMAS COMPORTAMENTAIS
}

\author{
PSYCHOLOGY AND SCHOOL INCLUSION: NEW WAYS OF INTERVENING TO \\ PREVENT BEHAVIORAL PROBLEMS
}

\author{
Aline Maira da SILVA ${ }^{1}$ \\ Enicéia Gonçalves MENDES ${ }^{2}$
}

\begin{abstract}
RESUMO: no contexto da inclusão educacional, um papel que parece promissor para o psicólogo escolar é o de prestar Consultoria Colaborativa Escolar, atuando em parceria com professores e familiares. O objetivo do estudo foi avaliar os efeitos de um programa de intervenção preventiva, baseado nos modelo de Consultoria Colaborativa Escolar e Suporte Comportamental Positivo, voltado para prevenir e minimizar problemas comportamentais. O estudo foi realizado em três salas de aula do primeiro ano de uma escola municipal de ensino fundamental, localizada no interior do estado de São Paulo, e contou com a participação das três professoras das salas e seus 55 alunos. O estudo foi conduzido em quatro etapas. Na Etapa 1, foram realizados os procedimentos éticos. Na Etapa 2, foi aplicado o Inventário dos Comportamentos de Crianças e Adolescentes 6-18 anos/Relatório para Professores (TRF), junto às professoras. Na Etapa 3, o programa de intervenção preventiva foi implementado e teve como alvo as professoras e os alunos. Por fim, na Etapa 4, o TRF foi reaplicado. Para avaliar o impacto da intervenção, foi aplicado o teste MANOVA nos resultados obtidos. Em relação ao comportamento dos alunos, houve diminuição estatisticamente significativa nos comportamentos do tipo internalizante, externalizante e nos problemas totais. Esse resultado, além de indicar que a aplicação dos modelos de Consultoria Colaborativa Escolar e de Suporte Comportamental Positivo pode ser efetiva, aponta para o fato de que tais modelos podem ser utilizados por psicólogos escolares em intervenções preventivas voltadas para prevenir e minimizar problemas de comportamento na escola.
\end{abstract}

PALAVRAS-CHAVE: Educação Especial. Consultoria escolar. Problemas de comportamento. Inclusão educacional. Psicologia escolar.

\begin{abstract}
In an inclusive school environment, an important aspect of the role of educational psychologists that seems promising for educational psychologists is that of rendering Collaborative Consultation in Schools, in partnerships with teachers and families. The purpose of this study was to evaluate the effects of a preventive intervention program, based on the principles of the Collaborative Consultation in Schools and the Positive Behavior Support models, designed to prevent and minimize behavior problems. The study was conducted in three $1^{\text {st }}$ grade classrooms in a public elementary school located in the state of São Paulo, involving participation of three teachers of these classrooms and their fifty-five pupils. The study was carried out in four phases. In Phase 1, the ethical procedures were carried out. In Phase 2, teachers were requested to complete the Child and Youth Behavior Inventory (6-18 years)/Teacher Report Form (TRF). In Phase 3, the preventive intervention program was implemented, targeting and focusing on teachers and pupils. Finally, in Phase 4, the TRF instrument was repeated. In order to assess the impact of the intervention, the MANOVA test was applied to the results. Regarding student behavior, there was a statistically significant decrease in internalizing, externalizing problems, and in total problems. Besides indicating that the implementation of the Collaborative Consultation in Schools and the Positive Behavior Support models can be effective, this result points to the fact that these models can be used by educational psychologists in preventive interventions that target preventing and minimizing behavior problems in school.
\end{abstract}

KEYWORDS: Special Education. Collaborative Consultation in Schools. Behavior Problems. School inclusion. Educational psychology.

\footnotetext{
1 Doutora em Educação Especial pela Universidade Federal de São Carlos. Professora adjunta da Faculdade de Educação da Universidade Federal da Grande Dourados (FAED/UFGD). alinesilva@ufgd.edu.br

${ }^{2}$ Doutora em Psicologia. Professora adjunta do Departamento de Psicologia da Universidade Federal de São Carlos - Programa de Pós-Graduação em Educação Especial (PPGEEs/UFSCar). egmendes@ufscar.br
} 


\section{INTRODUÇÃo}

Muitos professores se queixam da indisciplina dos alunos, do descaso das famílias, e se declaram desamparados por não saberem como lidar com situações envolvendo problemas comportamentais. Os problemas comportamentais por sua vez podem interferir não só no aprendizado acadêmico, mas no desenvolvimento global dos estudantes.

Assim, uma das principais demandas dos professores e educadores em geral, direcionadas aos profissionais da Educação Especial tem sido de como lidar com classes heterogêneas e, particularmente, com alunos que apresentam problemas de comportamentos. Este tipo de queixa referente aos comportamentos desafiadores dos alunos é ainda mais contundente se o psicólogo é o profissional que vai a escola com a proposta de colaborar com os professores.

Tradicionalmente, os psicólogos escolares têm baseado sua prática em um modelo médico de prestação de serviço, com uma atuação baseada na avaliação, diagnóstico, encaminhamento e tratamento dos alunos a eles encaminhados. Criticando este modelo de atuação, Sheridan e Gutkin (2000) consideram que os psicólogos escolares deveriam estar menos preocupados em identificar o que está errado com o aluno, tentando mensurar os problemas e ofertando serviços remediativos. Para esses autores tais ações restringem a proposta de atuação da Psicologia Escolar, pois muito tempo é gasto para encontrar diagnósticos mais precisos, sendo tais informações pouco relevantes para a seleção e planejamento de intervenções educacionais efetivas.

Adicionalmente, os mesmos autores destacam a inadequação deste modelo médico em contextos escolares por pressupor que o trabalho deva ser realizado diretamente com o aluno, quando no entanto, professores, familiares, pares, entre outros, criam ambientes que podem favorecer ou suprimir o surgimento de comportamentos problemáticos, sendo portanto, necessário que essas pessoas sejam envolvidas na intervenção.

Paternite e Johnston (2005) consideram que devem ser criadas oportunidades para que profissionais, como o psicólogo, por exemplo, estabeleçam parcerias com educadores, compondo equipes interdisciplinares que tenham como objetivo focalizar a promoção de saúde, prevenção de problemas e a implementação de intervenções efetivas.

Um importante papel do psicólogo em ambientes escolares deve ser trabalhar de maneira colaborativa com familiares, professores e outros profissionais no delineamento de intervenções preventivas que melhorem a qualidade de vida dos alunos, promovendo o aprendizado e o desenvolvimento deles no ambiente escolar. Para tanto, psicólogos podem auxiliar os professores a diversificar e aprimorar sua postura ao ensinar, a manejar a sala de aula para diminuir comportamentos inapropriados apresentados pelos alunos, e ensinar- 
lhes habilidades sociais, além de auxiliar familiares no desenvolvimento de práticas parentais mais positivas.

Mas será que o psicólogo pode contribuir com sua prática para construir uma escola de melhor qualidade para todos os alunos indistintamente? A tese aqui defendida é de que a Psicologia Escolar tem muito a contribuir para a melhoria da escola para todos, embora como afirma Guzzo (2008), a intervenção do psicólogo escolar

[...] tal como tem sido caracterizada neste final do século, denota uma alienação na compreensão do que, efetivamente, acontece e não se traduz em mudança na realidade, pelo contrário penaliza o aluno que, neste contexto, é o objetivo final do sistema (GUZZO, 2008, p. 28)

Considerando o problema apontado, a proposta geral do presente estudo consistiu em investigar como delinear e testar experimentalmente uma proposta de atuação do psicólogo na escola. Com a finalidade de delimitar o estudo, buscamos circunscrever a proposta de intervenção aos problemas comportamentais dos alunos.

A ocorrência de problemas de comportamento é comumente observada nas escolas e ocasionam consequências negativas para professores, alunos e familiares. Familiares, professores, colegas e vizinhos, que fazem parte do ambiente dessa criança, podem arranjar condições que propiciam ou mantêm comportamentos indesejáveis. Dessa forma, problemas de comportamento não devem ser considerados simplesmente como ações inapropriadas das crianças, mas como interações inadequadas entre essas crianças e outras pessoas (KAUFFMAN, 2005).

Problemas comportamentais podem ser definidos, segundo Kauffman (2005), como uma necessidade educacional especial caracterizada por respostas comportamentais ou emocionais diferentes das respostas de uma idade apropriada, cultura ou normas éticas. Tais respostas afetam adversamente o desempenho educacional, incluindo habilidades sociais, vocacionais e pessoais.

Os problemas de comportamento podem ser descritos de acordo com duas dimensões: comportamento internalizante e comportamento externalizante. Segundo Gresham e Kern (2004), o comportamento internalizante refere-se a todos os comportamentos problema que são direcionados interiormente e que representam problemas que o indivíduo tem consigo mesmo, tais como: apresentar níveis de atividade baixo ou restrito, não falar com outras crianças, timidez, falta de assertividade, isolamento de situações sociais, preferência por ficar sozinho, agir de modo assustado, não participar de jogos e atividades, não apresentar respostas para interações sociais iniciadas por outras pessoas, não se posicionar, etc.

Por sua vez, os comportamentos externalizantes referem-se aos comportamentos inadequados que são direcionados para o ambiente social (GRESHAM; KERN, 2004). Segundo Kauffman (2005), comportamentos externalizantes são caracterizados por comportamentos antissociais que se referem 
a qualquer comportamento relacionado com a violação das regras sociais e/ou atos contra os outros, tais como, agressão, roubo, mentiras e vandalismo.

Definido como foco das intervenções os "problemas comportamentais" dos alunos, buscamos investigar na literatura algumas propostas de atuação dos psicólogos nas escolas e nesta busca encontramos duas abordagens que julgamos mais adequadas para os propósitos do estudo, a saber: Consultoria Colaborativa Escolar e Suporte Comportamental Positivo.

A Consultoria Colaborativa Escolar pode ser definida como um processo no qual um consultor trabalha em uma relação igualitária e não hierárquica com um consultante, em geral professores e pais, de forma a reunir os esforços dos mesmos para tomar decisões e implementar intervenções que melhor atendam os interesses educacionais dos alunos (KAMPWIRTH, 2003).

Dessa forma, por meio da Consultoria Colaborativa Escolar é possível tornar grupos de pessoas, com habilidades e conhecimento diversos, capazes de produzir soluções criativas para problemas definidos mutuamente. Um dos objetivos gerais no modelo de Consultoria Colaborativa é melhorar e manter as competências de todos os envolvidos, incluindo aqueles que apresentam necessidades educacionais especiais (IDOL; NEVIN; PAOLUCCI-WHITCOMB, 2000).

Investigações científicas têm sido realizadas com o intuito de avaliar percepções sobre o processo de Consultoria Colaborativa assim como avaliar os possíveis efeitos da aplicação desse modelo na escola.

Upshur, Wenz-Gross e Reed (2009) desenvolveram um estudo cujo objetivo foi avaliar um programa de intervenção, baseado na consultoria, dirigido para crianças consideradas em situação de risco, seus professores e pais. $\mathrm{O}$ estudo foi realizado em cinco escolas de educação infantil e contou com a participação de 37 professores, 47 familiares e 136 alunos, com idades entre três e cinco anos e identificados com problemas de comportamento do tipo externalizante. Dos 136 alunos, apenas 47 participaram da intervenção, sendo que os 89 alunos restantes não puderam participar devido a não autorização dos pais, e fizeram parte do grupo controle não equivalente.

O programa oferecido teve duração de três anos e incluiu: a) observação dos alunos em sala de aula; b) trabalho individual com a criança em sala de aula; c) sessões de terapia individual com a criança; d) consultoria com os pais visando orientá-los acerca de maneiras de disciplinar e apresentar regras aos filhos; e) consultoria com os professores visando orientá-los sobre como lidar com o comportamento dos alunos e prestar esclarecimentos sobre o comportamento e o desenvolvimento das crianças; f) mediação de encontros entre familiares e professores; e, g) orientação para os familiares sobre como procurar por outros recursos e serviços quando necessário.

A análise dos dados relacionados com as 47 crianças com comportamento externalizante e seus familiares, comparados com as 89 crianças do grupo 
controle e a análise dos dados da amostra de 19 crianças do grupo experimental e 19 do grupo controle, revelou que a intervenção foi associada com redução dos comportamentos agressivos, do comportamento não adaptativo e também com aumento na emissão de comportamento adaptativos.

Um estudo sobre a Consultoria Colaborativa realizado no Brasil por Alpino (2008) teve como objetivo verificar os efeitos de uma proposta de Consultoria Colaborativa promovida por uma fisioterapeuta junto às professoras de cinco alunos com paralisia cerebral. O estudo foi desenvolvido em cinco escolas municipais do sistema regular de ensino. A Consultoria Colaborativa foi efetivada por meio de intervenção direcionada para as professoras e para os alunos. A intervenção voltada para as professoras incluiu: a) planejamento colaborativo; b) palestras sobre deficiência física, paralisia cerebral e suas implicações educacionais; e, c) orientações especializadas às professoras participantes, em sala de aula. Por sua vez, a intervenção voltada para os alunos incluiu: a) indicação, desenvolvimento e implementação de adaptações do mobiliário e dos materiais escolares; b) acompanhamento dos alunos em sala de aula; e, c) orientações aos pais, por meio de visitas domiciliares.

Após a intervenção, foi observada melhora do alinhamento postural dos alunos participantes. As professoras relataram que houve melhora, por parte dos alunos, na segurança, autoestima, coordenação e interesse em participar das atividades e redução da necessidade de reposicioná-los na cadeira. Além disso, as professoras destacaram sentimento de maior segurança em relação ao atendimento educacional e assistência aos alunos com paralisia cerebral.

Pereira (2009) investigou as possibilidades da Consultoria Colaborativa do psicólogo no ambiente escolar que pudessem favorecer a inclusão escolar de alunos surdos. Participaram da pesquisa seis professoras de três classes comuns dos primeiros anos do ensino fundamental de uma escola pública. As professoras autorizaram a observação quinzenal de suas aulas pelo consultor e se reuniram quinzenalmente com o mesmo. As três salas contavam com um total de seis alunos surdos (G1) e a pesquisadora solicitou ainda às docentes que indicassem seis alunos com indicativos de comportamento socialmente aceito (G2) e outros seis com indicativos de problemas de comportamento (G3). Como medida de desempenho escolar, os alunos ouvintes e surdos participaram de um teste de desempenho escolar no início e final do ano de 2006, com a autorização de seus responsáveis.

Para verificação de habilidades prossociais e problemas de comportamento as professoras responderam escalas comportamentais a respeito dos alunos dos três grupos de estudo. As escalas comportamentais possibilitaram a discriminação de comportamentos socialmente aceitos e orientações de manejos comportamentais que contribuíram para o aumento de oportunidades para que os mesmos ocorressem. A avaliação de desempenho apontou para uma correlação entre problemas de comportamento e dificuldades de aprendizagem, além de indicar que práticas vigentes têm contribuído para o ensino de aritmética aos surdos, mas 
ainda não lhe garantiram, pelo menos nesta escola, a alfabetização, requisito este essencial para acessibilidade ao conhecimento escolar.

No presente estudo o modelo de Consultoria Colaborativa Escolar foi adotado em associação ao modelo de Suporte Comportamental Positivo (SCP).

O SCP tem sua origem na análise do comportamento e foi desenvolvido, inicialmente, como uma alternativa para intervenções aversivas utilizadas com alunos com graus severos de deficiência que se engajavam em formas extremas de agressão contra os outros e contra si mesmo (SUGAI et al., 1999). Recentemente, o SCP tem sido aplicado em contextos mais amplos, com o objetivo de promover comportamentos prossociais e minimizar problemas de comportamento (WARREN et al., 2006).

O SCP é um modelo de intervenção preventiva que visa alcançar mudanças comportamentais socialmente importantes (CARR et al., 1999). Para tanto, utiliza métodos educacionais baseados em procedimentos da análise do comportamento para ajudar indivíduos a desenvolver comportamentos socialmente apropriados por meio da mudança do sistema social no qual o indivíduo está inserido (CARR, 2007).

Dessa maneira, a atenção é focalizada na criação e manutenção de ambientes escolares que promovam qualidade de vida para todas as crianças e jovens, fazendo com que o comportamento problema se torne menos efetivo, eficiente e relevante e com que um comportamento mais funcional seja estimulado. Além disso, o uso de intervenções culturalmente apropriadas é enfatizado (SUGAI et al., 1999).

De acordo com a perspectiva do Suporte Comportamental Positivo o foco da intervenção não pode ser simplesmente reduzir a ocorrência do comportamento problemático. Por outro lado, a intervenção deve focalizar o aumento das habilidades relevantes e criar sistemas que ajudam a apoiar a satisfação pessoal e a felicidade. Por isso, as intervenções baseadas nesse modelo não fazem uso de procedimentos aversivos (WARREN et al., 2006; CARR, 2007).

O SCP tem se mostrado uma estratégia preventiva promissora para diminuir a emissão de comportamentos inadequados apresentados pelos alunos (RONES; HOAGWOOD, 2000).

Lier, Vuijk e Crijnen (2005) realizaram um estudo com o objetivo de verificar a eficácia de uma intervenção baseada no SCP, denominada como "Jogo do Bom Comportamento" - JBC ${ }^{3}$. O JBC envolveu uma intervenção preventiva implementada em sala de aula que teve como objetivo promover comportamentos socialmente adequados.

Participaram do estudo 666 crianças, com idade média de 6,9 anos, de 13 escolas municipais. As salas de aula foram distribuídas randomicamente entre o grupo controle e o grupo experimental. Das 31 classes participantes, 15 classes foram designadas como grupo controle e 16 classes como grupo experimental.

3 Tradução do termo inglês "Good Behavioral Game” (GBG). 
A aplicação do JBC envolveu três fases: fase introdutória, fase de expansão e fase de generalização. Na fase introdutória, o JBC foi jogado três vezes por semana durante, aproximadamente, dez minutos. Além dos elogios, as equipes receberam como recompensa figuras autoadesivas após cada jogo. Na fase de expansão, os professores foram encorajados a expandir a duração do JBC, ampliar os ambientes nos quais o JBC era jogado e aumentar o número de comportamentos alvo. Nessa fase, as recompensas foram adiadas para o final de cada mês. Finalmente, na fase de generalização, foi enfatizada a promoção de comportamentos socialmente adequados também quando o JBC não estava sendo aplicado. Para tanto, elogios como consequências para comportamentos adequados também foram utilizados pelos professores durante toda a aula. Como resultado, grandes reduções de comportamentos antissociais foram obtidas entre crianças do grupo experimental que apresentavam altos índices desses comportamentos. Tais reduções coincidiram com filiações com pares não desviantes e com diminuição da rejeição de pares.

Um estudo longitudinal foi conduzido por McClean et al. (2005) com o objetivo de avaliar a efetividade de uma intervenção, com base no SCP visando a redução de comportamentos desafiadores. Participaram do estudo 132 profissionais que prestavam serviços para os 138 clientes (adultos e crianças com diferentes níveis de dificuldades de aprendizagem) também participantes. Os profissionais receberam treinamento, com duração de seis meses, para fazer uso do SCP com os seus clientes.

Durante o treinamento, os profissionais foram instruídos a selecionar um comportamento alvo (comportamento desafiador) apresentado pelos seus clientes. Como parte do treinamento, cada comportamento escolhido foi especificado pelos profissionais com uma definição, sendo que tais definições foram compartilhadas pelos participantes. A frequência de emissão dos comportamentos alvo foi registrada pelos profissionais participantes continuamente, desde a linha de base até a fase de seguimento.

Como resultado, foi observado que $77 \%$ do grupo de profissionais relataram redução substancial na emissão de comportamentos desafiadores em relação aos dados coletados durante a linha de base, depois da implementação da intervenção. Em relação à porcentagem média de mudança comportamental, foi observada redução de $73,2 \%$ na fase de seguimento em comparação com os dados de linha de base.

Bradshaw et al. (2009) também desenvolveram um estudo com o objetivo de examinar o impacto de uma intervenção baseada no SCP. O estudo foi realizado em 37 escolas públicas e contou com a participação de 2.596 funcionários (1.437 professores e 1.159 profissionais da escola).

Do total de 37 escolas participantes, 21 foram submetidas à condição de intervenção (grupo intervenção) e 16 à condição de comparação (grupo comparação). Cada uma das escolas do grupo intervenção selecionou uma equipe de cinco ou seis pessoas entre professores e administradores para participar de 
um treinamento em SCP. Além do treino, as escolas do grupo de intervenção receberam suporte e assistência técnica contínua de treinadores.

O treino de SCP demonstrou ter efeito estatisticamente significativo no que diz respeito às seguintes variáveis: habilidade do diretor em obter recursos para a escola; relação entre os funcionários; e comportamento dos alunos.

Considerando as consequências negativas da ocorrência de problemas disciplinares nas escolas e os resultados promissores apontados pela literatura baseada em intervenções envolvendo a Consultoria Colaborativa Escolar e oSuporte Comportamental Positivo, o objetivo do estudo foi desenvolver, implementar e avaliar um programa de intervenção, voltado para prevenir problemas de comportamento, que combina os modelos de Consultoria Colaborativa Escolar e Suporte Comportamental Positivo.

\section{MÉTOdo}

$\mathrm{O}$ estudo desenvolvido envolveu delineamento experimental $\mathrm{AB}$, com pré-teste, intervenção e pós-teste e compreendeu quatro etapas: 1) Condução dos procedimentos éticos; 2) Coleta de medidas antes da intervenção (pré-teste); 3) Programa de intervenção preventiva baseada na CCE e no SCP, e 4) Coleta de medidas após a intervenção (pós-teste).

O estudo foi realizado em uma escola municipal de ensino fundamental localizada em um bairro da periferia de uma cidade de médio porte do interior do estado de São Paulo. Com a finalidade de melhor delimitar o estudo e tendo em vista o caráter preventivo que se queria imprimir à intervenção, definiu-se que o estudo seria dirigido para as professoras e os alunos do primeiro ano, que seria justamente a porta de entrada dos alunos na escola.

Assim, participaram do estudo as três professoras das três salas do primeiro ano do ensino fundamental da escola e os 55 alunos dessas salas, que frequentaram as aulas do início ao final da coleta de dados.

A idade das professoras variou entre 27 e 34 anos. A participante P1 tinha o menor tempo de experiência docente e era a única que ainda não havia concluído o curso de Pedagogia. As participantes P2 e P3 possuíam cursos de especialização, sendo que P3 tinha o maior tempo de experiência como docente.

Em relação aos alunos, participaram 36 meninas e 19 meninos, sendo 15 alunos da sala 1, 19 alunos da sala 2 e 21 alunos da sala 3. Os alunos apresentaram idades entre seis e sete anos. Um total de 64 alunos esteve presente durante a coleta de dados, mas não participaram de todas as etapas do estudo, pois deixaram a escola no final do primeiro semestre ou foram matriculados após o início da intervenção. Como esses alunos não participaram de toda a intervenção, os dados referentes a eles não foram considerados. Dessa forma, participaram de todas as etapas da pesquisa 55 alunos. 
Foi utilizado como instrumento o Inventário dos Comportamentos de Crianças e Adolescentes entre seis e 18 anos - Relatório para Professores/TRF (ACHENBACH; RESCORLA, 2001). O TRF avalia o funcionamento adaptativo e problemas de comportamento a partir de informações fornecidas pelos professores.

\subsection{Procedimento de coleta de dados}

$\mathrm{Na}$ Etapa 1 foi efetuado o contato com a escola e os procedimentos éticos foram conduzidos. Na Etapa 2 o TRF foi aplicado junto às três professoras participantes do estudo. Na Etapa 3 o programa de intervenção preventiva foi implementado e teve como alvo as professoras e os alunos participantes do estudo.

A intervenção com as professoras teve duração de sete meses e foi efetivada por meio de 19 reuniões semanais com duração aproximada de uma hora. Os temas das reuniões foram divididos em cinco módulos: conceitos (educação inclusiva, Consultoria Colaborativa Escolar, Suporte Comportamental Positivo e problemas de comportamento); modificações no ambiente físico da sala de aula; manejo de relacionamento; manejo de conduta; manejo de conteúdo (KARAGIANNIS, STAINBACK; STAINBACK, 1999; KAMPWIRTH, 2003; KAUFFMAN, 2005).

Entre as reuniões, as professoras receberam visitas, previamente agendadas, da pesquisadora com o objetivo de auxiliar na implementação das intervenções planejadas assim como discutir com cada professora sobre o desenvolvimento da intervenção em sua sala.

Considerando a importância do desenvolvimento de habilidades sociais na infância para prevenção e também minimização de problemas comportamentais, foi realizado treino de habilidades sociais com os alunos das três salas de aula alvo do estudo. $O$ treino foi realizado em cada uma das salas de aula, uma vez por semana, com duração aproximada de 50 minutos.

O conteúdo do treino de habilidades sociais foi desenvolvido com base em Del Prette e Del Prette (2005) e as sessões foram planejadas com o objetivo de englobar as sete classes de habilidades sociais que, segundo os autores, são prioritárias no desenvolvimento interpessoal da criança: autocontrole e expressividade emocional; habilidades de civilidade; empatia; assertividade; solução de problemas interpessoais; fazer amizade; e habilidades sociais acadêmicas.

Na Etapa 4, após a realização da intervenção, o TRF foi novamente aplicado com o objetivo de verificar os efeitos da intervenção.

\subsection{Procedimento de ANÁlise de dAdos}

Os escores obtidos com a aplicação do TRF foram analisados por meio do software Assessment Data Manager -ADM, que permite que os dados do inventário sejam digitados e analisados de acordo os parâmetros do instrumento. 
O software apresenta o escore total alcançado por cada aluno, assim como o escore $T$ e o percentil do escore total. A análise foi realizada considerando seis aspectos abordados pelo inventário: perfil das Síndromes, comportamento internalizante, comportamento externalizante, problemas totais, desempenho acadêmico e comportamento adaptativo.

Quanto ao perfil das Síndromes, o instrumento permitiu a avaliação de oito escalas: ansiedade/depressão; isolamento/depressão; queixas somáticas; problemas sociais; problemas de pensamento; problemas de atenção; comportamento de quebrar regras; comportamento agressivo.

No perfil das Síndromes, escores $T$ maiores ou iguais a 70 são classificados na categoria clínica, escores $T$ entre 65 e 69 são classificados na categoria limítrofe, indicando um número de problemas que causam preocupação, e escores $T$ abaixo de 65 são classificados na categoria normal. Nas escalas comportamento internalizante, comportamento externalizante e problemas totais, escores T acima de 63 são incluídos na categoria clínica, entre 60 e 63 na categoria limítrofe e abaixo de 60 na categoria normal (LABORATÓRIO DE TERAPIA COMPORTAMENTAL/USP, 2006).

Por fim, em relação ao desempenho acadêmico e ao comportamento adaptativo, escores $T$ menores que 30 indicam categoria clínica, que sugere clara necessidade de ajuda para melhorar as competências; entre 30 e 40 categoria limítrofe, o que sugere provável necessidade de ajuda para fortalecer determinadas competências, e maior que 40 indicam categoria normal. $\mathrm{O}$ comportamento adaptativo é medido no TRF por quatro escalas: engajamento nas atividades, comportamento apropriado, aprendizado e grau de felicidade (LABORATÓRIO DE TERAPIA COMPORTAMENTAL/USP, 2006).

Para comparar os dados obtidos por meio do TRF nas etapas de pré e pósteste, foi utilizado o teste de MANOVA por meio do aplicativo SPSS for Windows.

\section{Resultados}

Os resultados serão apresentados segundo os seis aspectos investigados pelo inventário: perfil das Síndromes, comportamento internalizante, comportamento externalizante, problemas totais, desempenho acadêmico e comportamento adaptativo. Cabe destacar que para análise dos dados foram considerados os dados brutos obtidos por meio da aplicação do TRF.

\subsection{Perfil das Síndromes}

Como pode ser observado na Tabela 1, foi possível observar diminuição estatisticamente significativa em seis escalas: ansiedade/depressão $(\mathrm{F}(59,645 ; 54)$ $=12,39 \mathrm{p}<0,001)$; isolamento/depressão $(\mathrm{F}(9,309 ; 54)=4,89 \mathrm{p}<0,05)$; problemas sociais $(\mathrm{F}(25,536 ; 54)=17,03 \mathrm{p}<0,001)$; problemas de atenção $(\mathrm{F}(519,282 ; 54)=$ 
$31,71 \mathrm{p}<0,001)$; comportamento de quebrar regras $(\mathrm{F}(7,645 ; 54)=6,57 \mathrm{p}<0,05)$; comportamento agressivo $(\mathrm{F}(62,627 ; 54)=13,59 \mathrm{p}<0,001)$.

Tabela 1 - Escalas do perfil das Síndromes no pré e no pós-teste.

\begin{tabular}{|c|c|c|c|c|c|c|}
\hline \multirow{2}{*}{ Escalas } & \multicolumn{2}{|c|}{ Pré-teste } & \multicolumn{2}{|c|}{ Pós-teste } & \multicolumn{2}{|l|}{ Manova } \\
\hline & média & $\begin{array}{l}\text { desvio } \\
\text { padrão }\end{array}$ & média & $\begin{array}{l}\text { desvio } \\
\text { padrão }\end{array}$ & $\mathrm{F}$ & $\mathrm{gl}$ \\
\hline Ansiedade/depressão & 2,16 & 3,06 & 0,69 & 1,39 & $12,39 * * *$ & $1 ; 54$ \\
\hline Isolamento/depressão & 1,38 & 2,24 & 0,80 & 1,13 & $4,89^{*}$ & $1 ; 54$ \\
\hline Queixas somáticas & 0,36 & 0,93 & 0,27 & 0,73 & ns & ns \\
\hline Problemas sociais & 1,44 & 2,06 & 0,47 & 0,9 & $17,03^{* * *}$ & $1 ; 54$ \\
\hline Problemas de pensamento & 0,22 & 0,63 & 0,11 & 0,37 & ns & ns \\
\hline Problemas de atenção & 9,22 & 8,5 & 4,87 & 5,05 & $31,71^{* * *}$ & $1 ; 54$ \\
\hline $\begin{array}{l}\text { Comportamento de } \\
\text { quebrar regras }\end{array}$ & 1,18 & 1,67 & 0,65 & 1,14 & $6,57^{*}$ & $1 ; 54$ \\
\hline $\begin{array}{l}\mathrm{Co} \mathrm{m} \mathrm{p} \mathrm{o} \mathrm{r} \mathrm{t} \text { a m e } \mathrm{nto} \\
\text { agressivo }\end{array}$ & 2,93 & 4,76 & 1,42 & 2,89 & $13,59 * * *$ & $1 ; 54$ \\
\hline
\end{tabular}

Nota: ${ }^{*} \mathrm{p}<0,05 ;{ }^{* * *} \mathrm{p}<0,001 ; \mathrm{ns}=$ não apresenta diferenças estatisticamente significativas.

\subsection{COMPORTAMENTO INTERNALIZANTE, EXTERNALIZANTE E PROBLEMAS TOTAIS}

Segundo a Tabela 2, houve diminuição estatisticamente significativa nas três escalas, comportamento internalizante $(F(126,582 ; 54)=12,24 \mathrm{p}<0,001)$, externalizante $(\mathrm{F}(114,036 ; 54)=12,75 \mathrm{p}<0,001)$ e problemas totais $(\mathrm{F}(2602,045 ; 54)=$ $32,62 \mathrm{p}<0,001)$.

Tabela 2 - Escalas comportamento internalizante, comportamento externalizante e problemas totais no pré e no pós-teste.

\begin{tabular}{lcccccc}
\hline & \multicolumn{2}{c}{ Pré-teste } & \multicolumn{2}{c}{ Pós-teste } & \multicolumn{2}{c}{ Manova } \\
\cline { 2 - 7 } escalas & média & $\begin{array}{l}\text { desvio } \\
\text { padrão }\end{array}$ & média & $\begin{array}{l}\text { desvio } \\
\text { padrão }\end{array}$ & F & Gl \\
\hline $\begin{array}{l}\text { Comportamento } \\
\text { internalizante }\end{array}$ & 3,91 & 4,71 & 1,76 & 2,33 & $12,24^{* * *}$ & $1 ; 54$ \\
\hline $\begin{array}{l}\text { Comportamento } \\
\text { externalizante }\end{array}$ & 4,11 & 5,93 & 2,07 & 3,72 & $12,75^{* * *}$ & $1 ; 54$ \\
\hline Problemas totais & 19,36 & 17,39 & 9,64 & 10,32 & $32,62^{* * *}$ & $1 ; 54$ \\
\hline
\end{tabular}

Nota: ${ }^{* *} \mathrm{p}<0,001$ 
No que diz respeito ao comportamento internalizante, o Quadro 1 apresenta os dados referentes aos alunos que tiveram mudanças de classificação entre o pré e o pós-teste. Conforme pode ser observado no quadro, o comportamento de quatro alunos (A30, A34, A40 e A42) foi enquadrado na categoria limítrofe e três alunos (A17, A59 e A60) na categoria clínica, antes da intervenção. Após a intervenção, com exceção de A42, cujo comportamento se manteve na categoria limítrofe, todos os demais alunos apresentaram diminuição nos escores e o comportamento dos mesmos passou a ser categorizado como normal. No caso, de A42, embora não tenha havido mudança na categorização, houve diminuição de dois pontos no escore.

Quanto aos demais alunos que participaram da intervenção, nenhum apresentou escore $T$ igual ou maior que 60 antes ou depois da intervenção e, por esse motivo, não estão representados no Quadro 1.

Quadro 1 - Escores e classificações dos comportamentos internalizantes no pré e pós-teste.

\begin{tabular}{|c|c|c|c|c|}
\hline \multicolumn{5}{|c|}{ COMPORTAMENTO INTERNALIZANTE } \\
\hline \multirow{2}{*}{ aluno } & \multicolumn{2}{|c|}{ pré } & \multicolumn{2}{|c|}{ pós } \\
\hline & escore $\mathrm{T}$ & categoria & escore $\mathrm{T}$ & categoria \\
\hline A17 & 75 & clínica & 45 & normal \\
\hline A30 & 62 & limítrofe & 50 & normal \\
\hline A34 & 62 & limítrofe & 44 & normal \\
\hline A40 & 60 & limítrofe & 44 & normal \\
\hline A42 & 62 & limítrofe & 60 & limítrofe \\
\hline A59 & 64 & clínica & 45 & normal \\
\hline A60 & 69 & clínica & 56 & normal \\
\hline
\end{tabular}

Quanto ao comportamento externalizante, é possível observar no Quadro 2 os escores obtidos pelos alunos que apresentaram mudança de classificação entre o pré e o pós-teste. Como mostra o quadro, seis alunos (A20, A30, A40, A48, A56 e A59) tiveram seu comportamento classificado na categoria limítrofe e seis alunos na categoria clínica (A12, A14, A15, A43, A47 A58).

Depois da intervenção, apenas uma aluna (A57), antes classificada na categoria normal, apresentou aumento nos escores e, consequentemente, teve seu comportamento classificado na categoria limítrofe. Embora os escores alcançados pelos alunos A12, A56 e A58 tenham diminuído depois da intervenção, essa diminuição não foi suficiente para alterar a classificação dos comportamentos apresentados por eles, que continuaram a ser enquadrados nas categorias clínica, limítrofe e clínica, respectivamente. Por outro lado, o comportamento de nove alunos (A14, A15, A20, A30, A40, A43, A47, A48 e A59) passou a ser classificado na categoria normal, após a intervenção. 
Quadro 2 - Escores e classificações dos comportamentos externalizantes no pré e pós-teste.

\begin{tabular}{|c|c|c|c|c|}
\hline \multicolumn{5}{|c|}{ Comportamento Externalizante } \\
\hline \multirow{2}{*}{ Aluno } & \multicolumn{2}{|c|}{ pré } & \multicolumn{2}{|c|}{ pós } \\
\hline & escore $T$ & categoria & escore $\mathrm{T}$ & categoria \\
\hline A12 & 67 & clínica & 66 & clínica \\
\hline A14 & 67 & clínica & 54 & normal \\
\hline A15 & 68 & clínica & 43 & normal \\
\hline A20 & 62 & limítrofe & 55 & normal \\
\hline A30 & 63 & limítrofe & 59 & normal \\
\hline A40 & 60 & limítrofe & 57 & normal \\
\hline A43 & 65 & clínica & 57 & normal \\
\hline A47 & 66 & clínica & 59 & normal \\
\hline A48 & 62 & limítrofe & 59 & normal \\
\hline A56 & 62 & limítrofe & 60 & limítrofe \\
\hline A57 & 59 & normal & 61 & limítrofe \\
\hline A58 & 68 & clínica & 66 & clínica \\
\hline A59 & 62 & limítrofe & 55 & normal \\
\hline
\end{tabular}

\subsection{DESEMPENHO ACADÊMICO E COMPORTAMENTO ADAPTATIVO}

Em relação ao desempenho acadêmico, segundo a Tabela 3, não houve mudança estatisticamente significativa. Por sua vez, quanto ao comportamento adaptativo, houve aumento estatisticamente significativo apenas na escala comportamento apropriado $(\mathrm{F}(1,782 ; 54)=5,93 \mathrm{p}<0,05)$.

Tabela 3 - Desempenho acadêmico e comportamento adaptativo no pré e pós-teste.

\begin{tabular}{lcccccc}
\hline & \multicolumn{2}{c}{ Pré-teste } & \multicolumn{2}{c}{ Pós-teste } & \multicolumn{2}{c}{ Manova } \\
\cline { 2 - 7 } escalas & média & $\begin{array}{c}\text { desvio } \\
\text { padrão }\end{array}$ & média & $\begin{array}{l}\text { desvio } \\
\text { padrão }\end{array}$ & F & gl \\
\hline $\begin{array}{l}\text { Desempenho } \\
\text { acadêmico }\end{array}$ & 2,94 & 0,8 & 3,05 & 0,87 & ns & $\mathrm{ns}$ \\
$\begin{array}{l}\text { Engajamento nas } \\
\text { atividades }\end{array}$ & 4,05 & 0,80 & 4,24 & 0,82 & $\mathrm{~ns}$ & $\mathrm{~ns}$ \\
$\begin{array}{l}\text { Comportamento } \\
\text { apropriado }\end{array}$ & 3,85 & 0,68 & 4,11 & 0,9 & $5,93^{*}$ & $1 ; 54$ \\
$\begin{array}{l}\text { Aprendizado } \\
\begin{array}{l}\text { Grau de felicidade } \\
\hline\end{array}\end{array}$ & 3,95 & 0,97 & 4,15 & 1,08 & $\mathrm{~ns}$ & $\mathrm{~ns}$ \\
\hline
\end{tabular}

Nota: ${ }^{*} \mathrm{p}<0,05 ; \mathrm{ns}=$ não apresenta diferenças estatisticamente significativas. 


\section{Discussão}

No que diz respeito à avaliação do impacto da intervenção no comportamento dos alunos, observou-se diminuição estatisticamente significativa em seis das oito escalas que fazem parte do perfil das síndromes avaliado pelo TRF: ansiedade/depressão; isolamento/depressão; problemas sociais; problemas de atenção; comportamento de quebrar regras; e comportamento agressivo. Também foi observada diminuição estatisticamente significativa nas escalas referentes ao comportamento externalizante, comportamento internalizante e problemas totais.

Resultados semelhantes foram encontrados em estudos internacionais desenvolvidos por Upshur, Weiz-Gross e Reed (2009), Lier, Vuijk e Crijnen (2005), McClean et al. (2005) e Bradshaw et al. (2009).

No estudo de Upshur, Weiz-Gross e Reed (2009), cujo objetivo foi avaliar um programa de intervenção, com base na Consultoria Colaborativa Escolar, voltado para crianças consideradas em situação de risco, os professores e os pais dessas crianças, a análise de dados revelou diminuição estatisticamente significativa na emissão de comportamentos agressivos e comportamento não adaptativo para o grupo controle. Mudanças significativas não foram observadas no comportamento dos alunos pertencentes ao grupo controle. Além disso, foi observado aumento estatisticamente significativo de comportamentos adaptativos.

Quanto aos comportamentos adaptativos, no presente estudo o instrumento TRF permitiu avaliar esse item investigando a percepção das professoras referente a três aspectos: engajamento nas atividades, comportamento apropriado, aprendizado e grau de felicidade. Foi observada mudança estatisticamente significativa apenas quanto ao comportamento apropriado.

Lier, Vuijk e Crijnen (2005), em estudo desenvolvido com o objetivo de verificar a eficácia de uma intervenção baseada no Suporte Comportamental Positivo, também verificaram reduções significativas de comportamentos antissociais nas crianças incluídas no grupo experimental.

O estudo longitudinal conduzido por McClean et al. (2005) com o objetivo de avaliar a efetividade de um modelo de prestação de serviços, baseado no Suporte Comportamental Positivo, voltado para indivíduoscom comportamentos desafiadores, teve como principal resultado que $77 \%$ do grupo de profissionais relataram redução substancial na emissão de comportamentos desafiadores, como efeito da intervenção.

Também foi observada melhora estatisticamente significativa no comportamento dos alunos que participaram do estudo de Bradshaw et al. (2009), cujo objetivo foi examinar o impacto de uma intervenção baseada no Suporte Comportamental Positivo. De acordo com os autores, após a intervenção, verificou-se que os alunos estavam mais cooperativos na sala de aula, respeitosos com os colegas e dispostos a melhorar suas habilidades. Essa mesma observação foi feita pelas professoras participantes do presente estudo, que avaliaram que todos os seus alunos se tornaram mais solidários e companheiros uns com os outros, após a intervenção. 
Cabe destacar que nenhum dos estudos acima citados investigou o impacto de intervenções baseadas na Consultoria Colaborativa Escolar e/ou no Suporte Comportamental Positivo no comportamento internalizante dos alunos. No presente estudo, houve a preocupação de planejar intervenções voltadas para prevenir e minimizar tais comportamentos, sendo que os resultados revelaram diminuição estatisticamente significativa em duas das três síndromes que estão relacionadas com problemas internalizantes: ansiedade/depressão e isolamento/depressão.

Quanto aos estudos nacionais que investigaram o efeito de programas de intervenções baseadas na Consultoria Colaborativa Escolar (ALPINO, 2008; PEREIRA, 2009), resultados positivos também foram observados. Alpino (2008) verificou o efeito de uma proposta de Consultoria Colaborativa Escolar implementada por uma fisioterapeuta junto às professoras de alunos com paralisia cerebral. Embora o estudo não tenha tido como foco problemas de comportamento apresentados pelos alunos, é importante destacar que mudanças comportamentais significativas foram observadas nos alunos alvo da pesquisa, no que diz respeito à autoestima e ao interesse em participar das atividades. No presente estudo não foram observadas mudanças estatisticamente significativas quanto ao comportamento dos alunos relacionado com o engajamento nas atividades e a autoestima não foi medida pelo instrumento utilizado.

Pereira (2009) desenvolveu um estudo com o objetivo de investigar as possibilidades de Consultoria Colaborativa do psicólogo no ambiente escolar para favorecer a inclusão escolar de alunos surdos. Apesar das dificuldades encontradas para estabelecer o processo de consultoria, Pereira (2009) concluiu que o psicólogo pode contribuir no contexto escolar, principalmente no que diz respeito ao manejo de comportamentos que visam à aprendizagem social dos alunos, sejam eles surdos ou não, favorecendo condições para o desempenho acadêmico.

Ao observar o impacto da intervenção para os alunos, é preciso discutir especificamente o caso de A57, que apresentou aumento de comportamentos inadequados do tipo externalizante, e os casos de A12, A42, A56 e A58 que não apresentaram melhoras tão evidentes.

A aluna A57 foi a única das três turmas que antes da intervenção foi classificada na categoria normal e, após a intervenção, na categoria limítrofe, quanto aos comportamentos do tipo externalizante. Essa mudança de classificação deveu-se ao aumento nos escores dos seguintes itens: desrespeitar regras, discutir excessivamente e envolver-se em brigas. Esse aumento pode ser em parte explicado pelo fato da aluna ter sofrido um acidente e ter se ausentado da escola durante três meses. Dessa forma, a aluna deixou de participar de grande parte do treino de habilidades sociais e das estratégias desenvolvidas em sala de aula. Além disso, depois desse período fora da escola e do convívio com os seus colegas de classe, são esperadas dificuldades em se adaptar à rotina da sala de aula, em se relacionar com os colegas e até mesmo dificuldades acadêmicas, que estão relacionadas com os comportamentos que passaram a ser emitidos pela aluna. 
Quatro alunos, embora tenham apresentado diminuição na emissão de comportamentos inadequados, não apresentaram melhoras significativas no comportamento. O comportamento do aluno A42, quanto ao tipo internalizante, se manteve na categoria limítrofe após a intervenção. Por sua vez o comportamento do tipo externalizante dos alunos A12 e A58 se manteve na categoria clínica e do aluno A56, permaneceu na categoria limítrofe, após a intervenção. Nestes casos, possivelmente terapias individualizadas poderiam ser tentadas.

\section{Conclusões}

O objetivo do estudo de desenvolver, implementar e avaliar um programa de intervenção voltado para prevenir problemas de comportamento, combinando os modelos de Consultoria Colaborativa Escolar e Suporte Comportamental Positivo na realidade brasileira foi alcançado.

Após análise e discussão dos resultados, é importante indicar caminhos para investigações futuras. Em primeiro lugar, consideramos a necessidade de investigar os efeitos da aplicação da Consultoria Colaborativa Escolar e do Suporte Comportamental Positivo, tendo como foco outras situações problema, por exemplo, as dificuldades de aprendizagem que também são uma queixa recorrente entre familiares e professores.

Também acreditamos que outros conteúdos possam fazer parte do programa de intervenção, no que diz respeito às estratégias desenvolvidas em conjunto com os professores, assim como às dinâmicas oferecidas no treino de habilidades sociais, de acordo com as diferentes realidades escolares existentes.

Há também a necessidade de realizar um estudo aplicando o programa de intervenção desenvolvido com alunos de outras faixas etárias. Dessa forma, com as devidas adaptações, o programa poderia ser voltado para alunos do primeiro ao quinto ano, seus professores e familiares. Consideramos que, ao envolver os professores do primeiro ao quinto ano, os efeitos da intervenção serão ampliados, já que, ao passar de um ano para o outro, os alunos encontrarão professores que darão continuidade ao trabalho de manejo eficiente de sala de aula desenvolvido no ano anterior. Com isso as modificações realizadas deixam de ser localizadas e passam a fazer parte do ambiente escolar, sendo incorporadas à cultura daquela escola.

Apesar da necessidade de estudos futuros investigarem os efeitos da aplicação da Consultoria Colaborativa e do Suporte Comportamental Positivo focalizando outras situações problema, outras faixas etárias e outros conteúdos para as intervenções, é possível afirmar que o presente estudo contribuiu para avançar o conhecimento quanto à forma como os modelos citados podem ser aplicados no Brasil.

Em vista disso, acreditamos que o presente estudo pode contribuir não apenas oferecendo uma proposta de programa de intervenção para ser implementada nas escolas brasileiras, mas também com um alerta de que muito precisa ser feito, 
em termos de investimento nos prédios escolares, valorização e capacitação de professores e, principalmente, contratação e qualificação de profissionais que os tornem capazes de desenvolver relações de parceria colaborativa com professores e familiares, visando atender à diversidade presente nas escolas e, consequentemente, promovendo o desenvolvimento de todos os alunos.

\section{REFERÊNCIAS}

ACHENBACH, T.M.; RESCORLA, L.A. Manual for the ASEBA School-Age form \& profiles. Burlington, VT: University of Vermont, Research Center of Children, Youths and Families, 2001.

ALPINO, A. M. S. Consultoria colaborativa escolar do fisioterapeuta: acessibilidade e participação do aluno com paralisia cerebral em questão. Tese (Doutorado em Educação Especial) - Universidade Federal de São Carlos, São Carlos, 2008.

BRADSHAW, C. P. et al. Altering school climate through school-wide positive behavioral interventions and supports: findings from a group-randomized effectiveness trial. Prevention science, v. 10, n. 2, 100-115, 2009.

CARR, E. G. et al. Positive behavior support for people with developmental disabilities: a research synthesis. Washington: American Association on Mental Retardation, 1999.

CARR, E. G. The expanding vision of positive behavior support: research perspectives on happiness, helpfulness, hopefulness. Journal of positive behavior interventions, v.9, n.1, p.3-14, 2007.

DEL PRETTE, A.; DEL PRETTE Z. A. P. Psicologia das habilidades sociais na infância: teoria e prática. Petrópolis: Vozes, 2005.

GRESHAM, F. M.; KERN, L. Internalizing behavior problems in children and adolescents. In: RUTHERFORD, R. B. e cols. Handbook of research in emotional and behavioral disorders. New York: The Guilford Press, 2004.

GUZZO, R. S. L. Saúde psicológica, sucesso escolar e eficácia da escola: desafios do novo milênio para a psicologia escolar. In: DEL PRETTE, Z. A. P. (Org.). Psicologia escolar e educacional: saúde e qualidade de vida. 3. ed. Campinas: Editora Alínea, 2008. p. 25-42. IDOL, L.; NEVIN, A.; PAOLUCCI-WHITCOMB, P. Collaborative consultation. Texas: Proed, 2000.

KAMPWIRTH, T. J. Collaborative consultation in the schools: effective practices for students with learning and behavior problems. New Jersey: Pearson Education, 2003.

KARAGIANNIS, A.; STAINBACK, W.; STAINBACK, S. Fundamentos do ensino inclusivo. In: STAINBACK, S.; STAINBACK, W. Inclusão: um guia para educadores. Porto Alegre: Artes Médicas, 1999.

KAUFFMAN, J. M. Characteristics of emotional and behavioral disorders of children and youth. $8^{\text {th }}$ edition. New Jersey: Pearson Educational, 2005.

LABORATÓRIO DE TERAPIA COMPORTAMENTAL DO INSTITUTO DE PSICOLOGIA DA UNIVERSIDADE DE SÃO PAULO. Guia para profissionais da saúde mental sobre o Sistema Empiricamente Baseado do Achenbach (ASEBA). Tiragem de circulação interna. São Paulo, 2006. 
LIER, P. A. C; VUIJK, P.; CRIJNEN, A. A. M. Understanding mechanisms of change in the development of antisocial behavior: the impact of a universal intervention. Journal of abnormal child psychology, v.33, n.5, p.521-535, 2005.

MCCLEAN, B. et al. Person focused training: a model for delivering positive behavioral supports to people with challenging behaviors. Journal of intellectual disability research, v.49, n.5, p.340-352, 2005.

PATERNITE, C. E.; JOHNSTON, T. C. Rationale and strategies for central involvement of educators in effective school-based mental health programs. Journal of youth adolescence, v.34, n.1, p.41-49, 2005.

PEREIRA, V. A. Consultoria Colaborativa na escola: contribuições da Psicologia para inclusão escolar do aluno surdo. Tese de doutorado em Educação Especial Universidade Federal de São Carlos, São Carlos, 2009.

RONES, M.; HOAGWOOD, K. School-based mental health services: a research review. Clinical child family psychological review, n.3, p.223-241, 2000.

SHERIDAN, S. M., GUTKIN, T. B. The ecology of school psychology: examining and changing our paradigm for the 21st century. The school psychology review, v.29, n.4, p.485-502, 2000.

SUGAI, G. et al. Applying positive behavior support functional behavioral assessment in schools. Technical Assistance Guide 1, Version 1.4.3. Washington: Center on Positive Behavioral Interventions and Support, 1999.

UPSHUR, C.; WENZ-GROSS, M.; REED, G. A pilot of early childhood mental health consultation for children with behavioral problems in preschool. Early chidhood research quarterly, v. 24, p. 29-45, 2009.

WARREN, J. S. et al. School-wide positive behavior support: addressing behavior problems that impede student learning. Educational psychology review, v.18, p.187-198, 2006.

Recebido em: 01/02/2011

Reformulado em: 11/07/2011

Aprovado em: 15/10/2011 